\title{
Mid- infrared uncooled sensor for the identification of pure fuel, additives and adulterants in gasoline
}

\author{
María Maldonado ${ }^{\mathrm{a}, \mathrm{b}}$, Pilar Barreiro ${ }^{\mathrm{b}, 1}$, Raul Gutiérrez $^{\mathrm{a}}$, Germán Vergara ${ }^{\mathrm{a}}$

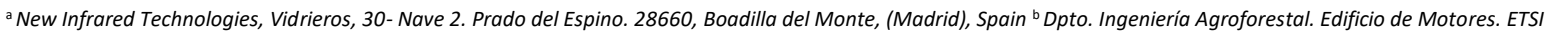 \\ Agrónomos. Universidad Politécnica de Madrid. Avda. Complutense, 3. Ciudad Universitaria. 28040, Madrid
}

\section{A B STR ACT}

\begin{abstract}
The aim of the present study is to test the ability of a low-cost and portable middle infrared spectrometer based on a linear array of $1 \times 128$ of PbSe, coupled with a linear variable

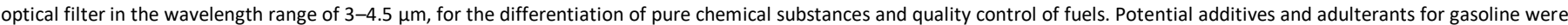

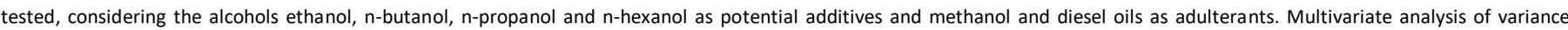

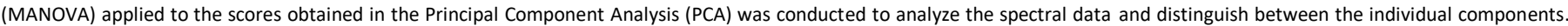

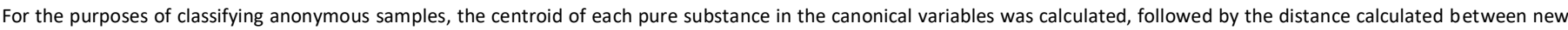

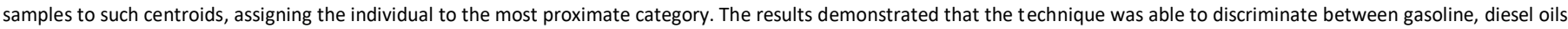
and the alcohols methanol, ethanol, n-propanol, n-butanol and $n$-hexanol and that it had the potential to be applied in the fuel industry.
\end{abstract}

\section{Introduction}

The means of transport currently in use are based on fossil fuels (petrol and diesel) which are non-renewable. Moreover, the increase in per capita energy use and improvements in the standard of living in developed and developing countries are enhancing this fuel dependency, with transport being a major contributor. In fact, the transport sector experienced an increase in the share of energy demand from $26 \%$ in 1990 to $32 \%$ in 2013 in the EU (Eurostat, simplified energy balance sheets, 2015 annual data) [9]. In recent years, fuel quality has become increasingly important, not only for its role in vehicle performance, but also for its impact on their emissions [21]. Fuel composition will continue to grow in importance as governments continue their strategy to achieve near-zero-emission vehicles. The removal of alkyl- lead compounds as an additive in gasoline, for environmental and public health reasons, has led oxygenated compounds to become increasingly important components in the formulation of automotive gasoline [3]. ETBE (ethyl tert-butyl ether) and alcohols (ethanol and butanol) are the oxygenated compounds most commonly added to gasoline $[8,17,18]$. Among the various alcohols, ethanol ( $100 \%$ renewable as bioethanol) is known as the most suitable, renewable, bio-based and eco-friendly fuel for spark-ignition engines $[5,10]$. In parallel, butanol has good prospects as gasoline blend stock $([10,17])$, since it presents better blending features compared to ethanol, but on the other hand some properties are better in ETBE. Nevertheless, it is not as renewable as alcohols $([5,10])$. These issues are shown in Table 1 . Higher chain alcohols (C3-C5) contain a high energy density, and are compatible with the current infrastructure as they are less hygroscopic [10]. These alcohols can be blended with gasoline. They also have the potential to be used as replacements, as butanol has already been shown to perform well in conventional gasoline engines $[10,17]$.

Normally fuels are adulterated by mixing them with comparatively lowerpriced adulterants which are chemically similar and necessarily easily miscible with them, such as industrial organic solvents, waste lubricants or kerosene [14]. Another kind of adulteration practice is frequent in countries where bioethanol is currently used as a common biofuel, mixed with gasoline in its dehydrated form (Hydrated Ethyl Alcohol Fuel, usually known as HEAF). HEAF is commonly adulterated with methanol, as methanol and ethanol present similar physical and chemical properties, and the price of methanol is lower than that of ethanol $[14,15]$. Fuel quality control has gained interest in many countries owing to the potential damage of low-quality fuel to engines, the environment, and economy. Thus, the application of analytical techniques to verify quality control of fuels has become crucial

Table 1

Gasoline blendstock comparison: Ethanol vs butanol and ETBE.

\begin{tabular}{|c|c|c|c|}
\hline PROPERTIES & ETBE & Ethanol & Butanol \\
\hline Blend octane $\mathrm{e}^{(1)}(\mathrm{R}+\mathrm{M}) / 2$ Blend & $1114-$ & 112 & 102 \\
\hline $\operatorname{RVP}^{(2)}(\mathrm{psi})$ & 6 & $18-22$ & $4-5$ \\
\hline Oxygen content (\%) & 15.66 & 34.7 & 21.6 \\
\hline Net energy (\% gasoline) & 84.347 & 65 & 82 \\
\hline Renewability ${ }^{(3)}(\%)$ & & 100 & 100 \\
\hline
\end{tabular}

$[4,6,11]$.

The fuel industry is constantly on the lookout for sensors to be used for insitu monitoring of fuel quality and adulteration detection. Vibrational spectroscopy techniques are non-destructive analytical techniques that allow reliable direct and fast determination of several properties at the same time without sample pre-treatment $([7,13])$. Infrared spectroscopy using Fourier transform spectrometers (FTIR) is the most common method used to determine the presence of adulterants in oils and petrol $[4,7,11]$. This is due to the fact that most organic compounds absorb radiation from the Infrared Spectrum [20].

The functional group region goes from 4000 to $1300 \mathrm{~cm}^{-1}$, and it offers general information about the chemical structure of a particular compound. The so-called fingerprint region goes from 909 to $650 \mathrm{~cm}^{-1}$ where the absorption of molecules belonging to the same functional group is quite different. The MWIR spectrometer used for this study works only in the functional region [20]. In previous studies, infrared spectroscopy has been used to predict the properties of ethanol as an additive in gasoline blending [12], and for raw ingredients in the production of rocket propellant, as well as for fuel quality control issues [16].

Mathematical analysis is used as a complementary tool for infrared spectroscopy, specifically multivariate techniques $([4,11])$. Nevertheless, FTIR spectrometers are expensive and heavy devices, which means that they are suitable for laboratory use but not for in situ applications which may require several devices installed along the product chain.

\footnotetext{
${ }^{1}$ Corresponding author.

E-mail address: pilar.barreiro@upm.es (P. Barreiro).
} 
For this reason there are plans to introduce low-cost spectrometers for some applications.

These have huge potential in systems for chemical analysis by optical absorption, fluorescence and emission line characterization [1]. Such micro spectrometers offer significant advantages over existing instruments, including size reduction, small sample size, and fast data acquisition [2]. However, on the down side, this kind of sensors have a limited signal-to noise ratio and poor resolution (in the case under study, the resolution is $60 \mathrm{~nm}$ ).

This disadvantage can be proven by the mathematical tools used in Chemometrics [4]. On the other hand, the infrared sensor used in this study is covered by a linear variable filter (LVF). This filter is based on a tapered cavity on top of a linear array of photodetectors, enabling the transfer of the optical spectrum into a lateral light intensity profile over the array of photodetectors. The same concept for this system can be designed and implemented for wavelengths ranging from UV to IR $(0.3-5 \mu \mathrm{m})$. In previous studies, LVF microspectrometers for UV and visible spectral ranges were presented [1,2].

The sensor used in this study provides a potential solution of portable equipment for fuel identification, using chemometrics as a support for the identification and segregation of gasoline, its additives (ethanol, propanol, butanol and hexanol) and two types of adulterants (methanol and diesel oil).

\section{Materials and methods}

The instrumentation and set-up consist of an infrared source, a sample chamber and a detector. The mid-infrared (MIR) spectrometer used for this study was based on a nondispersive linear sensor model CORE-HS LUXELL 128, which is manufactured by the company New Infrared Technologies (Boadilla del Monte, Madrid). It is made of vapor phase deposited PbSe (VPD PbSe) and has a coupled linear variable filter (LVF), in the wavelength range between 3 and $4.5 \mu \mathrm{m}$. The PbSe detection band ranges between 1 and $5 \mu \mathrm{m}$. The peak detection wavelength is $3.7 \mu \mathrm{m}$. The integration time ranges between 4 and $20 \mu \mathrm{s}$ (selectable on the instrument). The acquisition rate is up to 300 spectra per second [18], so it is faster than other kinds of sensors. This spectrometer, with no moving parts, is a rugged, compact and economically affordable device. An image of this sensor and the experimental set-up is displayed in the picture below (fig. 1). The infrared light source consisted of a SiC filament and a parabolic reflector (IR-SiX, Hawkeye Technologies, Inc.).

During the experiments, the intensity was set at $4.5 \mathrm{~A}$, while voltage was fixed at $6.5 \mathrm{~V}$. Liquid samples were allocated between two sapphire windows without any spacer in between, thereby allowing a very thin optical path (around $200 \mu \mathrm{m}$ ).

The $60 \mathrm{~nm}$ spectral resolution imposed by the spectrometer LVF + linear array performance levels means that chemometrics have to be used to reveal "hidden" information included in the low-resolution IR spectra obtained and, analyze the chemical species and extract their features.

Samples and chemicals.

The gasoline samples were taken in two different seasons (winter and summer gasoline according to legislation) and from different
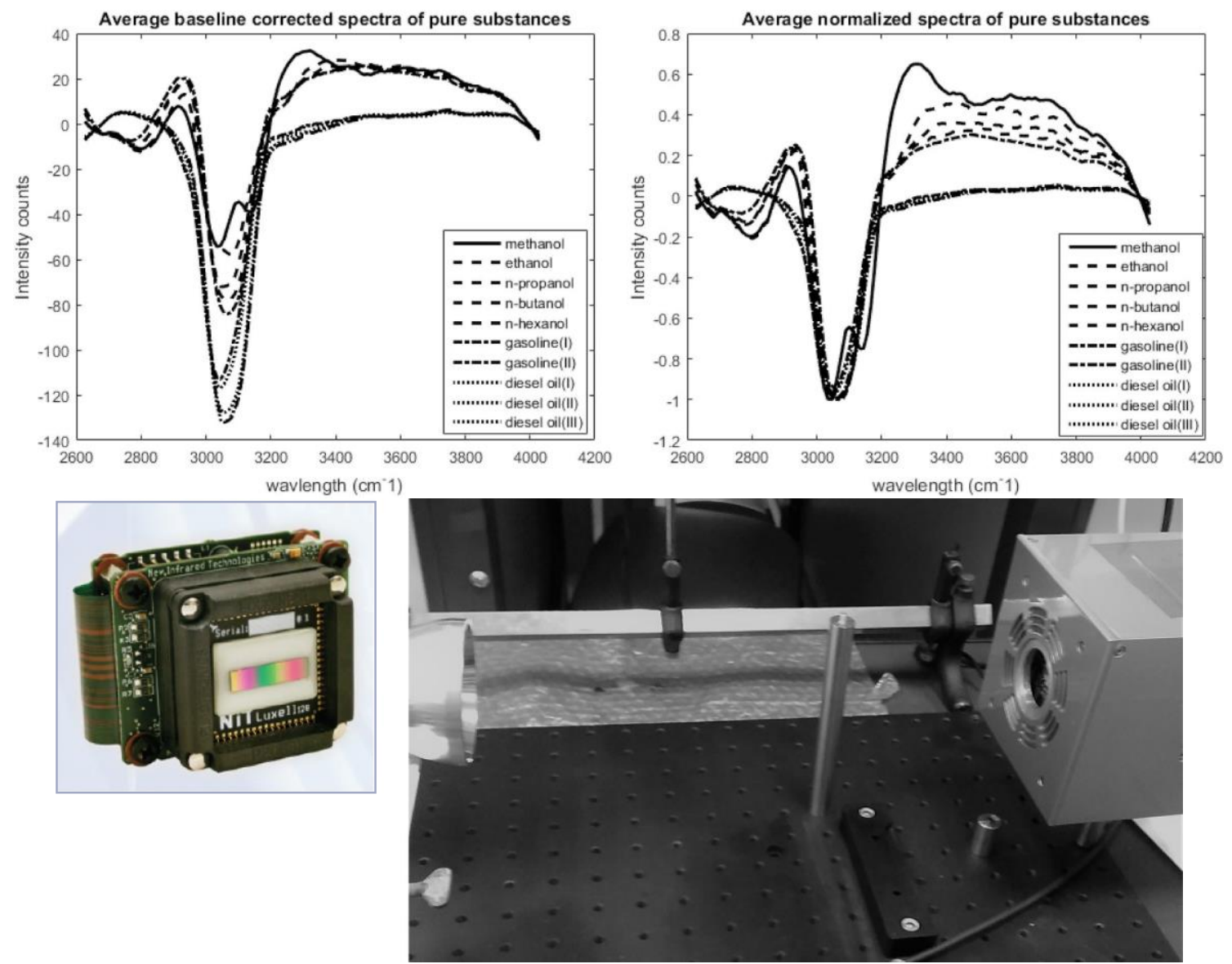

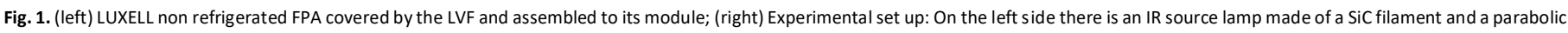

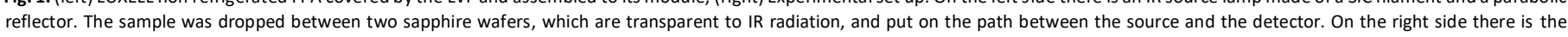
camera containing the IR detector made of PbSe with a coupled LVF.

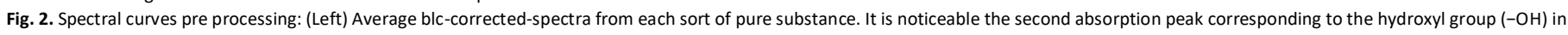
the methanol at $3145 \mathrm{~cm}^{-1}$ whilst the absorption peak of hydrocarbon chains corresponds to $3068 \mathrm{~cm}^{-1}$ in diesel oils and gasoline and moves to $3046 \mathrm{~cm}^{-1}$ in alcohols. 
(Right) Average normalized spectra from each of the pure substances. sources. They also had different octane numbers (between 95 and 98). Three diesel oil samples were used, also of different quality and origin. The chemicals used for the test were different long chained alcohols as laboratory reagents (99.5\% analytical grade). They were all bought from Panreac ${ }^{\circledR}$. The alcohols used were methanol, ethanol, n-propanol, n-butanol and n-hexanol.

\section{Measurements.}

Measurements were taken on different days to ensure the repeatability and reproducibility of the results. To be more precise, the alcohols were measured on seven different dates and the fuels were measured on nine different days.

\section{Calibration set.}

The calibration set was formed by 75 samples of different longchained alcohols (methanol, ethanol, propanol, n-butanol and hexanol), two sorts of gasoline (winter and summer), three sorts of diesel oils (different petrol stations). 3 replicates and 3 repeats were acquired from each fuel sample and 2 replicates and 3 repeats were acquired from each alcohol.

\section{Validation set.}

The validation set was formed by 420 samples of 3 replicates and 3 repeats acquired from measurements conducted on several days. These samples were of the same types of gasoline, diesel oils and alcohols. These data sets were concatenated and then projected onto the PC and MANOVA spaces. Projection onto the PC space was achieved by multiplying the normalized spectra by the loading matrix. The new canonical projections were obtained by first centering the PC scores of the validation set with regard to the calibration data, and subsequently projecting them onto MANOVA, via multiplication by the eigenvectors. Chemometric methods.

The MATLAB ${ }^{\circledR}$ computing environment (Mathworks, Inc.) was used for the mathematical analysis. The first step consisted of Principal Component Analysis. Afterwards, multivariate analysis of variance (MANOVA) was carried out by addressing the ability to distinguish between the additives and adulterants based on the PCA extracted from the MWIR spectra.

\section{Spectral pre-processing.}

The raw spectral curves acquired by the spectrometer were first smoothed in order to remove the noise, using the Savitzsky-Golay filter [20]. Baseline correction was then conducted on them by means of a Matlab function (Barreiro et al., 2016) who averages the curves from two ranges of the spectra. The baseline is the straight line that joins these two averages. The main aim of the baseline correction is correcting the drift of the device for it not to affect the results. Afterwards, a normalization of the curves was performed to the maximum absorption value, in order to remove the influence of intensity through the analysis.

\subsubsection{Principal component analysis (PCA).}

Principal Component Analysis was conducted on the pre-processed spectral data. It is useful to select a reduced set of PCs according to their relative weight for the non-supervised analysis. Since the PCA subspace was defined by the calibration set, the maximum number of PCs could not be more than 75 , retaining the information contained in 128 wavelengths. Once the PC variables are defined, any new set of spectra (in this case the validation set formed by 420 spectra) may be projected on this subspace.

\section{Discriminant analysis (MANOVA).}

One-way multivariate analysis of variance was applied to the PC scores obtained from the calibration set, using a categorical variable labelled according to the description of the materials and methods provided in the first section: gasoline, additives and adulterants. Canonical variables are a linear combination of the PCs that are created in this analysis, and they retain the maximum ability for segregation between individuals grouped in each category. Since MANOVA is preferably computed over a reduced amount of independent variables, in the case of MIR spectra, it is convenient to apply PCA in advance. MANOVA works by means of the diagonalization of Wilks's lambda matrix (in-between group variance compared to within-group or intragroup variance). The contribution of the original wavelengths to the canonical variables can be computed as $\mathrm{X}$ (normalized spectra)*L (loading matrix) *EV (eigenvectors matrix).
After screening the quality of segregation done by MANOVA, a classification procedure was implemented by computing the Euclidean distance of each sample in the validation set (420 spectra) from the centroid of the categories in the canonical space created from the PCs of the calibration set (75 spectra). This procedure is then available for any new anonymous sample under routine analysis.

\section{Results and discussion}

\section{Spectra of pure substances and absorption wavelengths.}

Fig. 2 shows the average baseline-corrected spectra for all types of gasoline (winter and summer), diesel oils (adulterant type 2), additives (ethanol, npropanol, n-butanol and n-hexanol), and adulterant type 1 (methanol), and also the average normalized spectra for these same samples. The theoretical absorption peaks for each type of bond that is present in fuels and alcohols are indicated in Table 2 [14]. In all five average spectra, the main absorption band was found to be around $3000 \mathrm{~cm}^{-1}$, with slight changes in spectra shape, also referred as spectral fingerprint. Fig. 3 plots the complete set of 495 raw spectra Table 2

Theoretical absorption wavelengths for hydrocarbons bonds. These hydrocarbons are present on the two kinds of gasoline (winter and summer), diesel oil (adulterant type 2), ethanol (additive) and methanol (adulterant type 1), in the mid infrared range used for this study (2222.22-3333.33 $\left.\mathrm{cm}^{-1}\right)$ [20].

\begin{tabular}{ll}
\hline Wavelength/wavenumber $\left(\mathbf{c m}^{-\mathbf{1}}\right)$ & Chemical functional group stretching \\
\hline $2850-3000$ & Alkanes $(\mathrm{CeH})$ \\
$3000-3100$ & Aromatic $(\mathrm{CeH})$ \\
$3080-3140$ & Alkene $(\mathrm{CeH})$ \\
$3300-3320$ & Alkyne $(\mathrm{CeH})$ \\
$3300-3600$ & Alcohol $(-\mathrm{OH})$ \\
\hline
\end{tabular}

(alcohol on the left, and gasoline and diesel oil on the right). A large vertical dispersion is found, blurring the clarity of the averaged spectra and pointing to the need for chemometric techniques for the individual assignment of samples.

\section{Principal Component Analysis.}

The variance percentage retained by the first five principal components accounts for more than $99.9 \%$ of the total variance from the normalized spectra. ANOVA analysis was performed on these principal components, pointing out that $\mathrm{PC} 2$ and $\mathrm{PC} 3$ have higher relevance than $\mathrm{PC} 1$. This may be due to the fact that PC1 usually gathers information related to the signal intensity, that should not be very different among samples from the same substance; while PC2 and PC3 contain information about other spectral features that may be more relevant for reducing the scale of the information. Fig. 4 (top left) displays the loadings or wavelength weights for PC 1, PC 2, PC 3 and PC 5 which provide the highest segregation of the individual spectra into fuel, additive and adulterant ( 1 and 2 ). The most relevant wavelengths are identified by the maximum absolute values in the loading plot: $3046 \mathrm{~cm}^{-1}$ (PC1); 2991, 3046 and $3156 \mathrm{~cm}^{-1}$ (PC5); $3068 \mathrm{~cm}^{-1}$ (PC2) and 2957, $3090 \mathrm{~cm}^{-1}$, $3167 \mathrm{~cm}^{-1}$ (PC3).

Although the highest percentage of the variance is retained by PC1 and PC2, the slight percentage retained by the PC5 $(0.07 \%)$ could result in a significant value as regards key spectral information. The scores of principal components 1 versus 2, 1 versus 3; and 1 versus 5 for fuels, additives and adulterants were represented in each Cartesian plane in Fig. 4 (top right, bottom left and bottom right respectively). The following remarks may be made:

PC1 (x-axis) allows to distinguish methanol from fuels, while not allowing such a clear separation between fuels, methanol and the rest of alcohols. Moreover, there is little specific spectral information contained in PC 1 , since there are no sharp bands in the load plot (Fig. 4, top left).

PC2 (y-axis) versus PC1 (x-axis), makes a linear function of signal intensity with the hydrocarbon chain length of substances.

PC3 ( $y$-axis) is able to difference between gasoline and diesel oils and also methanol from the rest of alcohols (additives).

PC5 (y-axis) is capable of differentiating between gasoline and diesel oils.

Classification procedure. 
In order to improve the segregation between alcohols, additives (ethanol, propanol, butanol or hexanol) and adulterants (methanol), a Multivariate Analysis of Variance was performed.

Multivariate Analysis of Variance (MANOVA).

MANOVA differs from ANOVA in that it combines the segregation ability of several different inputs ( $P C s$ in this case). The analysis is performed on the basis of PC $1-5$ (99.9\% of original spectral variance. Fig. 5 (left)) shows the canonical scores for the calibration and validation data sets, showing neat segregation of the pure substances in three main clusters: alcohols, diesel oils and gasoline. Methanol appeared to be clearly differentiated from the rest of the alcohols, due to its particular spectral curve, with the hydroxyl absorption peak more pronounced than in the others. The validation data are the result of projecting anonymous spectra onto the PC space and then onto the canonical space by multiplying the spectral matrix by the eigenvectors obtained from MANOVA, and thus confirming the repeatability and reproducibility of the analysis.

Fig. 5 (right) presents the wavelength loading for canonical variables 1 and 2 , retaining a segregation ability of $90 \%$ when all additives are considered separately, and $100 \%$ when gasoline, methanol, other alcohols, and diesel oils

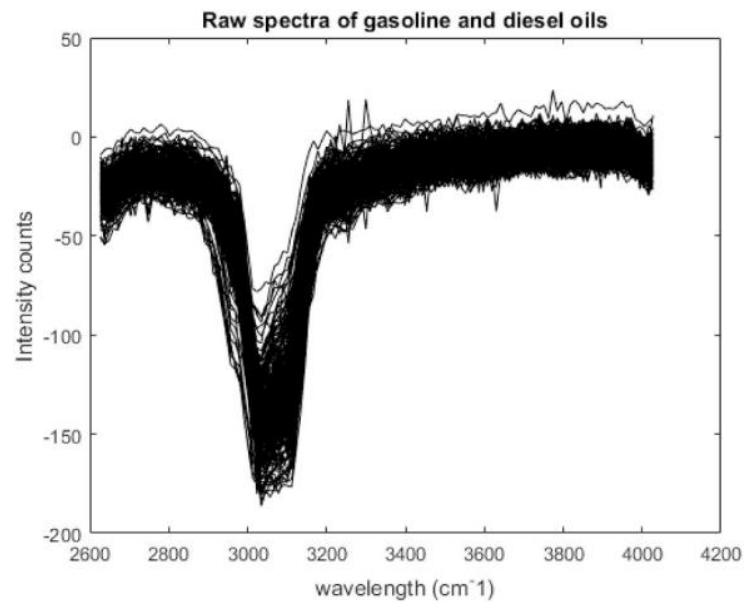

are considered - in other words, when ethanol, propanol, butanol and hexanol are considered together.

Characteristic values.

Table 3 provides the average canonical values for each sample in either the calibration or the validation set showing significant congruence (among similar samples) and robustness (repeatability over time, 9 days in the validation set besides calibration) in the procedure. Thus, the average differences between gasolines and diesel oils remain in the region of 34 and $36 \%$ for canonical 1 and 67.7 and $68.4 \%$ for canonical 2. On the other hand, MANOVA uses the highest wavelengths values (such as $3167 \mathrm{~cm}^{1}$ ) to segregate between alcohols, as these compounds have the higher absorption frequencies (see Table 2), and the lowest ones to segregate between diesel oils and gasoline, because according to the composition of gasoline, who is richer in alkenes and aromatics than diesel, it should have higher frequency values than diesel oils.

Unsupervised substance identification in the case of diesel additives and adulterants.

A classification algorithm was developed on the basis of calculating the distance between each individual in the canonical plane with regard to the centroids for each category: gasoline, additive (ethanol, propanol, butanol or hexanol), adulterant 1 (methanol) and adulterant 2

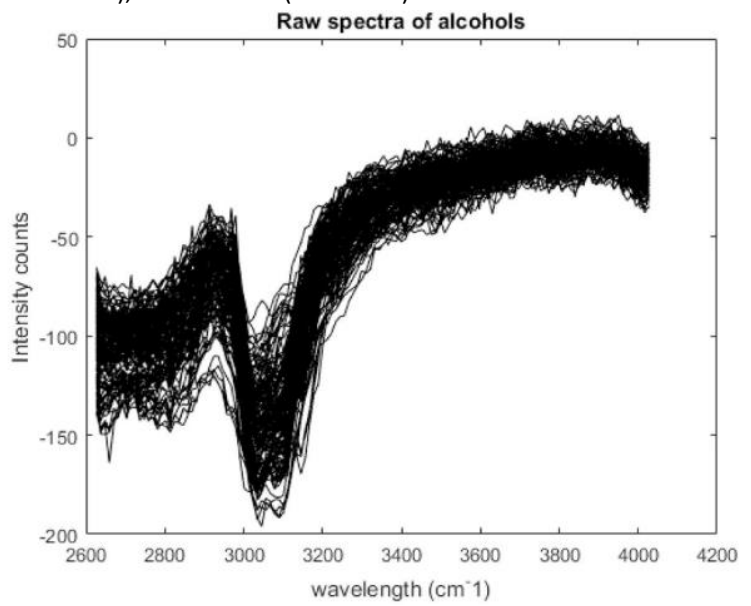

Figure 3. (Left) raw spectra of fuels (gasoline and diesel). Raw spectra of alcohols (Right). 

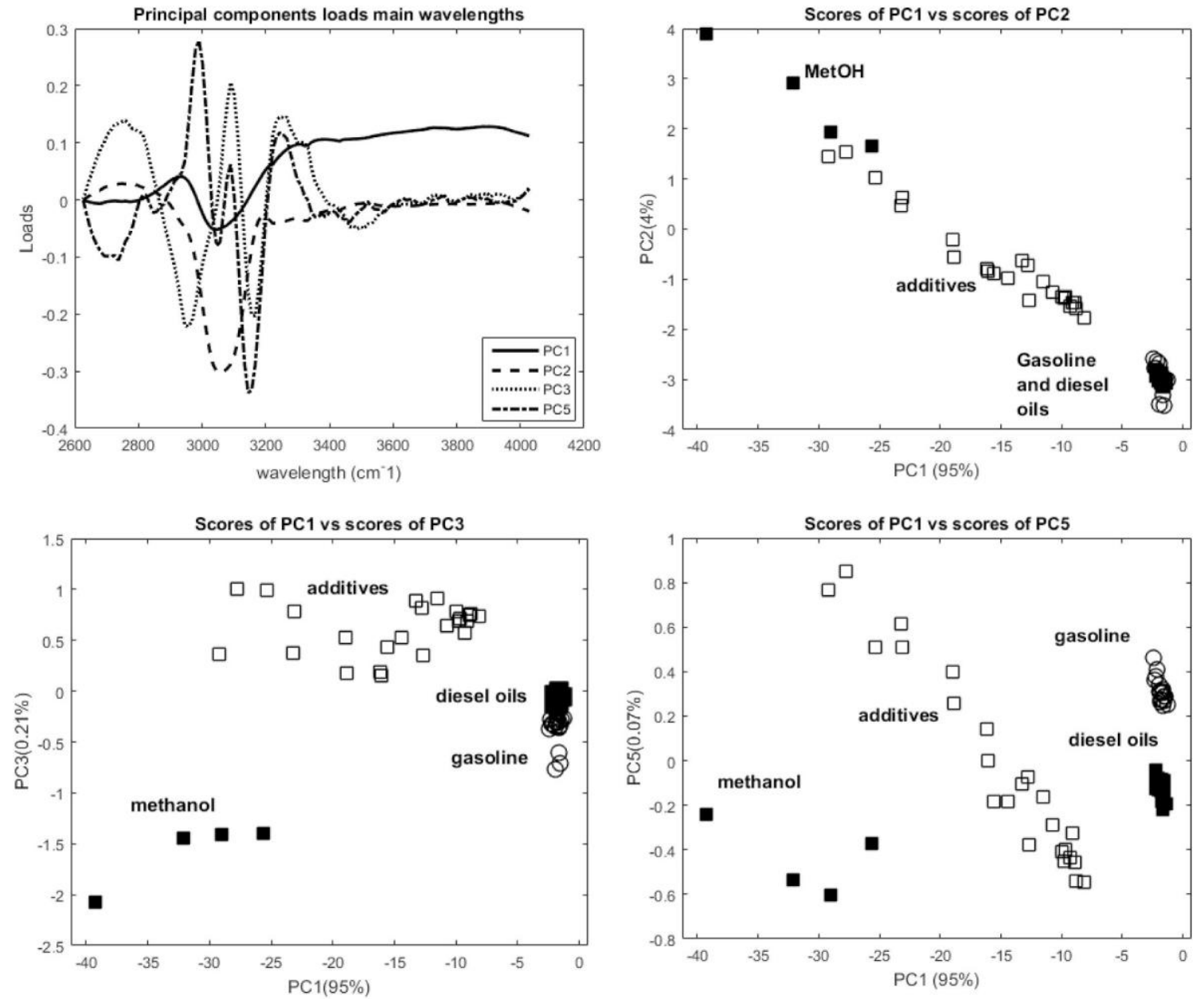

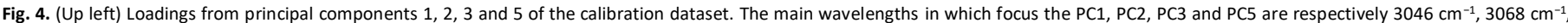

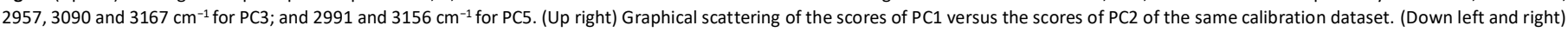
Graphical scattering of the scores of PC1 versus the scores of PC3 and PC5 respectively.
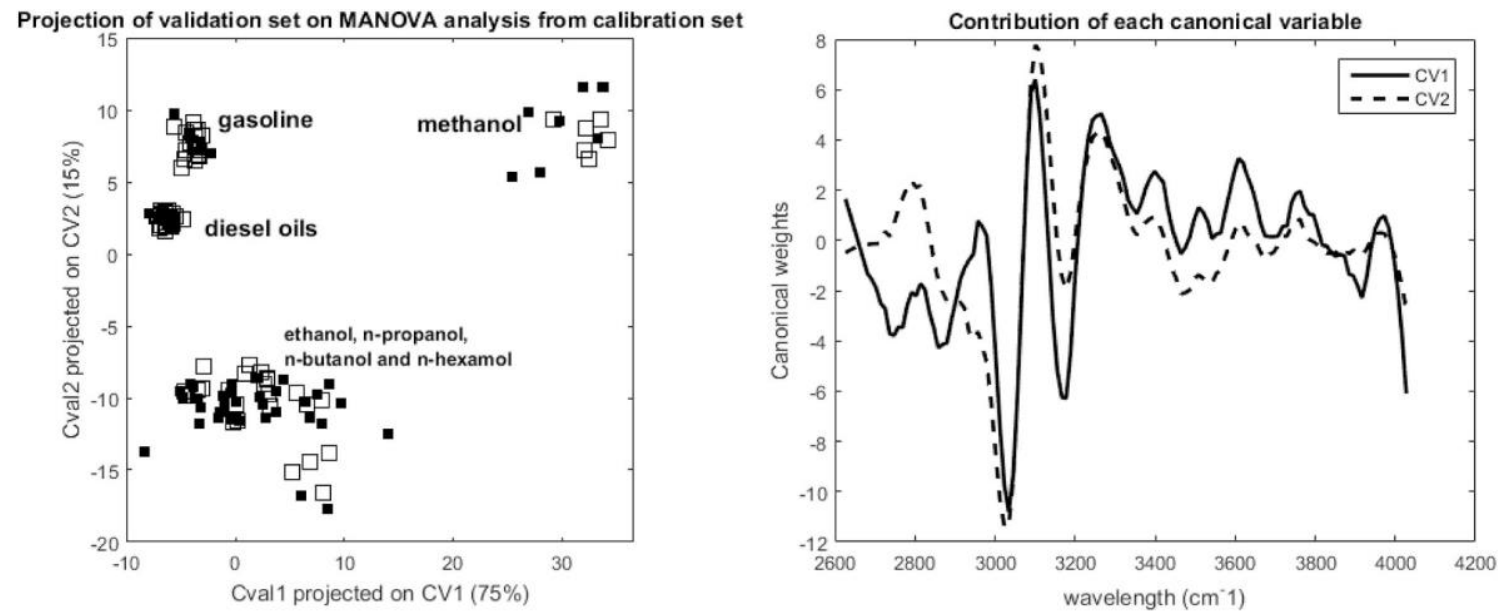

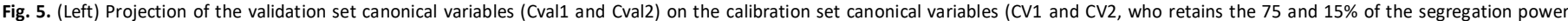

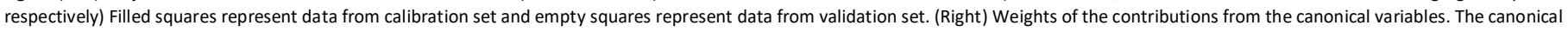
variable 1 focuses on three wavelengths of 3035 and $3101 \mathrm{~cm}^{-1}$ and the canonical 2 focuses on 3024 and $3112 \mathrm{~cm}^{-1}$. Both of them focus also in $3167 \mathrm{~cm}^{-1}$.

(diesel oil). Table 4 compares the observed and predicted classes for prospect for a fast, simple and reliable method for the in situ differboth the calibration and validation sets with $100 \%$ consistency. entiation of pure chemical substances that are potential adulterants or additives in fuels such as gasoline. The procedure was validated as re4. Conclusions gards the repeatability of the methods (tests conducted on several different dates).

This paper proposes an MWIR detector that provides a clear The identification of the key absorption wavelengths for each Table 3

Characteristic values from MANOVA. The Average values of each canonical variable from the calibration and validation set are displayed. 


\begin{tabular}{|c|c|c|c|c|c|}
\hline & & Canonical 1 & & Canonical 2 & \\
\hline & & Calibration & Validation & Calibration & Validation \\
\hline Potential adulterant (1) & Methanol & 32.31 & 29.48 & 8.19 & 8.42 \\
\hline \multirow[t]{3}{*}{ Potential adulterant (2) } & Diesel (1) & -6.29 & -6.36 & 2.35 & 2.15 \\
\hline & Diesel (2) & -6.27 & -5.96 & 2.72 & 2.74 \\
\hline & Diesel (3) & -6.32 & -6.20 & 2.22 & 2.27 \\
\hline \multirow[t]{3}{*}{ Potential additive } & Propanol & 3.25 & 2.38 & -8.98 & -9.13 \\
\hline & Butanol & 0.89 & -0.46 & -10.05 & -10.18 \\
\hline & Hexanol & -3.24 & -3.80 & -9.24 & -9.70 \\
\hline \multirow[t]{2}{*}{ Fuel } & Gasoline(1) & -3.98 & -4.04 & 8.28 & 8.13 \\
\hline & Gasoline(2) & -4.08 & -4.11 & 6.78 & 6.99 \\
\hline
\end{tabular}

Table 4

Predicted and observed distances from the validation set groups to the calibration set centroids.

\begin{tabular}{ll}
\hline Observed & Predicted \\
Validation (calibration)
\end{tabular}

\begin{tabular}{lllll} 
& Gasoline & Additive & Adulterant 1 & Adulterant 2 \\
\hline Gasoline & $110(18)$ & 0 & 0 & 0 \\
Additive & 0 & $120(24)$ & 0 & 0 \\
Methanol (Ad1) & 0 & 0 & $28(6)$ & 0 \\
Oil adulterant (Ad2) & 0 & 0 & 0 & $162(27)$ \\
\hline
\end{tabular}

chemical compound is the most important step before starting the mathematical procedure. In this study, the absorption bands identified match the chemical information obtained from the bibliography. The proposed MWIR device gathers at least five absorption bands corresponding to the main functional groups, i.e. the methyl, methylene and hydroxyl groups that are typical of organic molecules. This device has a clear ability for classification based on the presence of functional groups such as hydroxyl. Moreover, the shorter the hydrocarbon chain, the higher the influence of the hydroxyl, as may be seen in the case of methanol [7].

Principal Component Analysis focuses on the most significant wavelengths, i.e. the most intense wavelengths in the spectra $\left(3145,3046\right.$ and $\left.3068 \mathrm{~cm}^{-1}\right)$ to group the spectra variables in a space with reduced dimensions. On the other hand, MANOVA chooses the most discriminating wavelengths (those that are most different to the rest) regardless of their intensity.

Highly volatile substances revealed greater dispersion in measurements, as may be seen in the Cartesian diagrams, particularly methanol. This calls for a device capable of ensuring a uniform measurement procedure, by fixing the sample volume between the wafers, as an issue to be explored in subsequent studies.

(1) Blend octane index $(R+M) / 2$ is the average value between the $R$ (Research octane number, RON) and the M (Motor octane number, MON). (2) RVP (Reid vapor pressure). It is defined as the absolute vapor pressure exerted by a liquid at $37.8{ }^{\circ} \mathrm{C}$ as determined by the test method ASTM-D-323. (3) Renewability is defined as being a commodity or resource that is inexhaustible or replaceable by new growth. The percentage of renewability is taken from the bibliography $[5,17]$.

\section{Acknowledgements}

The Spanish Ministry of Economy (DI1406814) for providing the funding that enabled this research to be conducted by the company New Infrared Technologies and the University Politécnica of Madrid. References

[1] A. Emadi, H. Wu, S. Grabarnik, G. de Graaf, K. Hedsten, P. Enoksson, J.H.G. Correia, R.F. Wolffenbuttel, An UV Linear Variable Optical Filter-Based Micro-Spectrometer, Procedia Engineering, Volume 5, Eurosensor XXIV (2010) 416-419.

[2] A. Emadi, H. Wu, S. Grabarnik, G. de Graaf, R.F. Wolffenbuttel, CMOS-compatible LVOFbased visible microspectrometer, Proc. SPIE 7680 (2010) 1-6.
[3] ATC - Technical Committee of Petroleum Additive Manufacturers in Europe.

[4] M. Bassbasi, A. Hafid, S. Platikanov, R. Tauler, A. Oussama, Study of motor oil adulteration by infrared spectroscopy and chemometrics methods, Fuel 104 (2013) 798804.

[5] Michael R. Connor, James C. Liao, Microbial production of advanced transportation fuels in non-natural hosts, Curr. Opin. Biotechnol. 20 (3) (2009) 307-315.

[6] Correia, Radigya M., Domingos, Eloilson, Cáo, Vagne M., Araujo, Brenda R.F., Sena, Sthefany, Pinheiro, Layla U., Fontes, André M., Aquino, Luiz Felipe M., Ferreira, Ernesto.

[7] C., Filgueiras, Paulo R.; Romão, Wanderson. Portable near infrared spectroscopy applied to fuel quality control. Talanta, 2017; 176: 26-33.

[8] E.W. De Menezes, R. Cataluña, D. Samios, R. da Silva, Addition of an azeotropic ETBE/ethanol mixture in eurosuper-type gasolines, Fuel 85 (2006) 2567-2577.

[9] Directive 2009/30/EC of the European Parliament and of the Council of 23 April 2009 amending Directive 98/70/EC as regards the specification of petrol, diesel and gas-oil and introducing a mechanism to monitor and reduce greenhouse gas emissions and amending Council Directive 1999/32/EC as regards the specification of fuel used by inland waterway vessels and repealing Directive 93/12/EEC. Official J Eur Union 2009; 140:88-113.

[10] I. Doroshenko, V. Pogorelov, V. Sablinskas, Infrared Absorption Spectra of Monohydric Alcohols. Dataset Papers in, Chemistry (2013) 1-6.

[11] V. Gaydou, J. Kister, N. Dupuy, Evaluation of multiblock NIR/MIR PLS predictive models to detect adulteration of diesel/biodiesel blends by vegetal oil, Chemom. Intell. Lab. Syst. 106 (2) (2011) 190-197.

[12] Berenice Hernandez-Castillejos, Silva-Oliver, Guadalupe, Sánchez-Minero, Felipe, Ramirez-Jimenez, Edgar, Predicción de propiedades de mezclas gasolina-etanol mediante espectroscopía de infrarrojo, Avances en Ciencias e Ingeniería 4 (2013).

[13] Johannes Kiefer, Recent advances in the characterization of gaseous and liquid fuels by vibrational spectroscopy, Energies 8 (4) (2015) 3165-3197.

[14] Naveen Garg, Shashank Mohan, Amit Pal, R. S. Mishra. Fuel Adulteration, Problem and Mitigation Strategies: A Review. International Conference of Advance Research and Innovation (ICARI-2015).

[15] Neto, Álvaro C., Oliveira Emanuele C. S., Lacerda Valdemar, Castro Eustáquio V. R. Romão, Wanderson, Silva, Renzo C., Pereira, Roni G., Sten Tiago, Filgueiras Paulo R., Poppi, Ronei J. Quality control of ethanol fuel, Assessment of adulteration with methanol using 1H NMR, Fuel 135 (2014) 387-392.

[16] Quan Zou, Guodong Deng, Xiaode Guo, Wei Jiang, Fengsheng Li, A Green Analytical Tool for In-Process Determination of RDX Content of Propellant Using the NIR System, ACS Sustain. Chem. Eng. 1 (12) (2013) 1506-1510.

[17] D.E. Ramey, Butanol: the other alternative fuel, Proceedings of the 19th annual conference of the National Agricultural Biotechnology Council. Brookings (United States): South Dakota State University, NABC, 2007, pp. 137-147.

[18] Luis Miguel Rodríguez-Antón, Fernando Gutierrez-Martín, Yolanda Doce, Physical properties of gasoline, isobutanol and ETBE binary blends in comparison with gasolineethanol blends, Fuel 166 (2016) 73-78.

[20] Segneanu, Adina E.; Gozescu, loan Dabici, A.; Sfirloaga, P.; Szabadai, Z. Organic compounds FT-IR spectroscopy.

[21] K.S. Singal, P.B. Pundir, Diesel Fuel Quality and Particulate Emissions: An Overview SAE Paper 961185, (1996). 